\title{
HIV treatment decision making: high rate of revised treatment choices based upon different genotypic interpretation systems E Wolf*1, E Gersbacher ${ }^{1}$, M Vogel ${ }^{2}$, A Eberhard ${ }^{3}$, J Goelz ${ }^{4}$, Y Yazdanpanah ${ }^{5}$, J Rockstroh ${ }^{2}$, G Fätkenheuer ${ }^{6}$, S Mauss ${ }^{7}$, C Mayr ${ }^{8}$, H Knechten ${ }^{9}$, I Van Walle ${ }^{10}$, E Jaegel-Guedes ${ }^{3}$ and $\mathrm{H}_{\text {Jaeger }}{ }^{3}$
}

\author{
Address: ${ }^{1}$ MUC Research, Munich, Germany, ${ }^{2}$ University Bonn, Bonn, Germany, ${ }^{3}$ HIV Research and Clinical Care Centre, Munich, Germany, \\ ${ }^{4}$ Praxiszentrum Kaiserdamm, Berlin, Germany, ${ }^{5}$ Centre Hospitalier de Tourcoing, Tourcoing, France, ${ }^{6}$ University Cologne, Cologne, Germany, \\ ${ }^{7}$ Center for HIV and Hepatogastroenterology, Duesseldorf, Germany, ${ }^{8}$ Aerzteforum Seestrasse, Berlin, Germany, ${ }^{9}$ PZB Aachen, Aachen, Germany \\ and ${ }^{10}$ Virco BVBA, Mechelen, Belgium \\ * Corresponding author
}

from Ninth International Congress on Drug Therapy in HIV Infection

Glasgow, UK. 9-13 November 2008

Published: 10 November 2008

Journal of the International AIDS Society 2008, I I (SuppI I):PI 95 doi:I0.II86/I758-2652-I I-SI-PI 95

This abstract is available from: http://www.jiasociety.org/content/I I/SI/PI95

(c) 2008 Wolf et al; licensee BioMed Central Ltd.

\section{Background}

Before initiating or changing antiretroviral therapy (ART), treatment decisions should be guided by HIV resistance testing. Expert- and data-based interpretation systems for genotypic mutation patterns are different approaches to evaluate levels of drug resistance. Various interpretation systems have shown good correlation with virological outcomes. However, they can lead to discordant resistance classifications. To date, there are few prospective data on whether various interpretation systems lead to different treatment decisions.

\section{Methods}

Prospective study in HIV-patients (pts) requiring ARTchange due to virological failure and genotypic resistance to $\leq 1$ drug. The choice of the new regimen was a three-step approach. The physician's first choice was based on the local standard of care genotypic interpretation (SOC). Thereafter, choice could be adapted according to the virtual phenotype report (vircoType, VT). Final treatment decision was made at the patient's visit. Drug activity scores were calculated as genotypic sensitivity scores (GSS) acc. to SOC-interpretation and as continuous phenotypic SS (cPSS) acc. to VT. For new drugs, scores were set to 1 if interpretation was not available (e.g. RAL, MVC).

\section{Summary of results}

$\mathrm{N}=44$ pts were included. Resistance to drugs of 3, 2 or 1 class was detected in $25 \%, 57 \%$, and $18 \%$, respectively. Of $\mathrm{N}=44$ VT-based choices, 23 (52\%) were different from SOC-based choices. Differences were related to $\leq 2$ drug classes in 12, to only PI or NRTI in 11 pts. New drug classes were foreseen in 11 SOC-choices and in 12 VTchoices. $64 \%$ of VT-choices were identical to final choices compared to only $39 \%$ of SOC-choices. For SOC-based choices, mean GSS was 2.1, mean cPSS was 2.0. Mean increase in CPSS from SOC-based to both VT- and final choices, was 0.21 . Regarding only pts with different VTand SOC-based choices, mean cPSS-increase was 0.40 (p $<0.05$ ) with no further increase if final choice was again different. Mean number of drugs did not differ for SOCand VT-choices (3.4), but was slightly higher for final regimens (3.6). Mean cPSS per drug was 0.61 for SOCchoices, 0.67 for VT-choices and 0.64 for final regimens.

\section{Conclusion}

The use of calculated phenotypic information (VT) derived from genotypic mutation patterns led to revised treatment decisions in more than $50 \%$ of HIV pts. New treatment choices mostly consisted in different use of NRTI and PI. There was a trend to higher activity scores in the revised treatment choices. 